\title{
LEFSCHETZ DUALITY FOR INTERSECTION (CO)HOMOLOGY
}

\author{
MARTINTXO SARALEGI-ARANGUREN
}

\begin{abstract}
We prove the Lefschetz duality for intersection (co)homology in the framework of $\partial$-pesudomanifolds. We work with general perversities and without restriction on the coefficient ring.
\end{abstract}

Given an orientable $n$-dimensional $\partial$-compact pseudomanifold $X$, the following Lefschetz duality

$$
\mathcal{D}_{X}: H_{D \bar{p}}^{*}(X ; R) \rightarrow H_{n-*}^{\bar{p}}(X, \partial X ; R)
$$

has been proved in [10] (see also [15, 8]). In these papers, the coefficient ring is a field and the general perversity $\bar{p}$ verifies $\bar{p} \leq \bar{t}$, where $\bar{t}$ is the top perversity. The RHS is the intersection homology of $X$. The LHS is the intersection cohomology defined by using $D \bar{p}$-intersection cochains, where $D \bar{p}$ is the complementary perversity of $\bar{p}$. The operator $\mathcal{D}_{X}$ is the cap-product by an orientation class of $X$.

The hypothesis $\bar{p} \leq \bar{t}$ can be eliminated if we avoid the $\bar{p}$-allowable simplices contained in the singular part of $X$. This can be done by using the tame $\bar{p}$-intersection homology $\mathfrak{H}_{*}^{\bar{p}}(X ; R)$ (see [2, 8]) for which the Lefschetz duality becomes

$$
\mathcal{D}_{X}: H_{D \bar{p}}^{*}(X ; R) \rightarrow \mathfrak{H}_{n-*}^{\bar{p}}(X, \partial X ; R) .
$$

This is proved in 8 ( 8 (see also [10]), where the hypothesis $R$ is a field has been weakened by: $X$ is a locally $(D \bar{p}, R)$-torsion free $\partial$-pseudomanifold.

Unfortunately, this result does not extend to a general ring. For example, for the closed cone $X=\mathrm{c} \mathbb{R} \mathbb{P}^{3}$ we get $H_{\bar{p}}^{2}(X ; \mathbb{Z})=\mathbb{Z}_{2} \neq 0=\mathfrak{H}_{2}^{\bar{p}}(X, \partial X ; \mathbb{Z})$, where $\bar{p}=D \bar{p}$ is the perversity taking the value 1 on the apex of $X$. In order to eliminate the torsion condition we use, as it is introduced in 5] for the Poincaré duality, the blown-up intersection cohomology $\mathscr{H}_{\bar{p}}^{*}(X ; R)$ and we get the Lefschetz duality

$$
\mathcal{D}_{X}: \mathscr{H}_{\bar{p}}^{*}(X ; R) \rightarrow \mathfrak{H}_{n-*}^{\bar{p}}(X, \partial X ; R)
$$

without restrictions on the coefficient ring $R$ (see Theorem $\underline{\mathrm{A}}$ ). Since $\mathscr{H}_{\bar{p}}^{*}(X ; R) \cong$ $H_{D \bar{p}}^{*}(X ; R)$, in the case of locally $(D \bar{p}, R)$-torsion free $\partial$-pseudomanifolds (see [3, Theorem F]) then we observe that (2) generalizes the Lefschetz duality (11) of 8 .

Section 4 is devoted to the proof of this Theorem. The method of the proof uses the Poincaré duality established in [5] for pseudomanifolds (see also [4] by using sheaf theory). In the same way as a boundary manifold is not a manifold, the space $X$ is

Date: November 17, 2018.

2000 Mathematics Subject Classification. 55N33, 55M05, 57N80.

Key words and phrases. Intersection homology, Lefschetz duality.

The author would like to thank Daniel Tanr for many helpful suggestions and comments in the preparation of this work. 
not a pseudomanifold and Poincaré duality does not apply directly. Then we refine the stratification of $X$ in order to obtain a pseudomanifold $X^{\natural}$ (see [8] for another point of view). This is done in Section 1. The relationship between tame intersection homology (resp. blown-up intersection cohomology) of $X$ and that of $X^{\natural}$ is done in Section 2 (resp. Section 3).

The transformation of a $\partial$-pseudomanifold $X$ into a pseudomanifold $X^{\natural}$ by refining the stratification as well as the comparison of both associated intersection homologies, by modifying the perversity on $X^{\natural}$, has been done for the first time on 9 in the category of PL-pseudomanifolds. This is the key point of the work.

We fix for the sequel a commutative ring $R$ with unity. All (co)homologies in this work are considered with coefficients in $R$. For a compact topological space $X$, we denote by c $X=X \times[0,1] / X \times\{0\}$ the closed cone on $X$ and $\stackrel{\circ}{ } X=X \times[0,1[/ X \times\{0\}$ the open cone on $X$. A point of a cone is denoted by $[a, t]$. The apex of these cones is $\mathrm{v}=[-, 0]$.

\section{Contents}

1. Pseudomanifolds

2. Tame intersection homology

3. Blown-up cohomology

4. Lefschetz Duality

References

\section{Pseudomanifolds}

We present in this section the geometrical objets used in this work.

Definition 1.1. A filtered space is a Hausdorff topological space endowed with a filtration by closed sub-spaces

$$
\emptyset=X_{-1} \subseteq X_{0} \subseteq X_{1} \subseteq \ldots \subseteq X_{n-1} \subsetneq X_{n}=X .
$$

The formal dimension of $X$ is $\operatorname{dim} X=n$.

The non-empty connected components of $X_{i} \backslash X_{i-1}$ are the strata of $X$. Those of $X_{n} \backslash X_{n-1}$ are regular strata, while the others are singular strata. The family of strata of $X$ is denoted by $\mathcal{S}_{X}$ or simply $\mathcal{S}$. The singular set is $X_{n-1}$, denoted by $\Sigma_{X}$ or simply $\Sigma$. The formal dimension of a stratum $S \subset X_{i} \backslash X_{i-1}$ is $\operatorname{dim} S=i$. The formal codimension of $S$ is $\operatorname{codim} S=\operatorname{dim} X-\operatorname{dim} S$.

Definition 1.2. A subset $U$ of $X$ can be provided with the induced filtration, defined by $U_{i}=U \cap X_{i}, i \in\{0, \ldots, n\}$. If $M$ is a topological manifold, with boundary or not, the product filtration defined by $(M \times X)_{i}=M \times X_{i}, i \in\{0, \ldots, n\}$.

When $X$ is compact, the cone filtration of cones $\stackrel{\circ}{ } X$ and $c X$ are defined respectively by $(\stackrel{\circ}{\mathrm{c} X})_{i}=\stackrel{\circ}{\mathrm{c}} X_{i-1}$ and $(\mathrm{c} X)_{i}=\mathrm{c} X_{i-1}, i \in\{0, \ldots, n+1\}$. We set $\stackrel{\circ}{\emptyset}=\mathrm{c} \emptyset=\mathrm{v}$, the apex of the cones $\stackrel{\circ}{\mathrm{X}}$ and $\mathrm{c} X$. In the closed cone $\mathrm{c} X$ we also consider the following filtration

$$
\left(\{\mathrm{v}\} \cup X_{0}\right) \subset \cdots \subset\left(\mathrm{c} X_{i-1} \cup X_{i}\right) \subset \cdots \subset \mathrm{c} X,
$$


$i \in\{1, \ldots, n\}$, denoted by $(\mathrm{c} X)^{\natural}$. The induced filtrations on $X$ by the filtrations $\mathrm{c} X$ and $c X^{\natural}$ are the same: the original filtration of $X$. Notice that we have the equalities: $(\mathrm{c} X)^{\natural} \backslash X=\stackrel{\circ}{ } X=(\mathrm{c} X) \backslash X$, as filtered spaces.

The more restrictive concept of stratified space provides a better behavior of the intersection (co)homology with regard to continuous maps.

Definition 1.3. A stratified space is a filtered space verifying the following frontier condition: for any two strata $S, S^{\prime} \in \mathcal{S}_{X}$ such that $S \cap \overline{S^{\prime}} \neq \emptyset$ then $S \subset \overline{S^{\prime}}$.

The relation $S \preceq S^{\prime}$, defined on the set of strata by $S \subset \overline{S^{\prime}}$, is an order relation (see [1. Proposition A.22]). The notation $S \prec S^{\prime}$ means $S \preceq S^{\prime}$ and $S \neq S^{\prime}$.

Definition 1.4. Let $f: X \rightarrow Y$ be a continuous map between two stratified spaces. The map $f$ is a stratified map, if it sends a stratum $S$ of $X$ on a stratum $S^{f}$ of $Y, f(S) \subset S^{f}$, verifying $\operatorname{codim} S \geq \operatorname{codim} S^{f}$.

When $f$ is a homeomorphism we say that $f$ is a stratified homeomorphism if $f, f^{-1}$ are stratified maps.

Notice that any inclusion 1: $Y \hookrightarrow X$ is a stratified map if we endow $Y$ with the induced filtration and $\operatorname{dim} Y=\operatorname{dim} X$. This last condition is equivalent to $Y \not \subset \Sigma_{X}$. The induced stratification is $\mathcal{S}_{Y}=\left\{(S \cap Y)_{c c} \neq \emptyset / S \in \mathcal{S}_{X}\right\}$, where ${ }_{c c}$ denotes a connected component.

Pseudomanifolds, introduced by Goresky and MacPherson in [11, 12], are filtered spaces having a conical local structure.

Definition 1.5. A n-dimensional pseudomanifold is a filtered space,

$$
\emptyset \subset X_{0} \subseteq X_{1} \subseteq \cdots \subseteq X_{n-2} \subseteq X_{n-1} \subsetneq X_{n}=X,
$$

such that, for each $i, X_{i} \backslash X_{i-1}$ is a topological manifold of dimension $i$ or the empty set. Moreover, for each point $x \in X_{i} \backslash X_{i-1}, i \neq n$, there exist

(i) an open neighborhood $V$ of $x$ in $X$, endowed with the induced filtration,

(ii) an open neighborhood $U$ of $x$ in $X_{i} \backslash X_{i-1}$,

(iii) an $(n-i-1)$-dimensional compact pseudomanifold $L$ where the open cone, $\stackrel{\circ}{\circ}$, is provided with the cone filtration,

(iv) a homeomorphism, $\varphi: U \times \stackrel{\circ}{\mathrm{c}} L \rightarrow V$, such that

(a) $\varphi(u, \mathrm{v})=u$, for each $u \in U$,

(b) $\varphi\left(U \times \stackrel{\circ}{\mathrm{c}} L_{j}\right)=V \cap X_{i+j+1}$, for each $j \in\{0, \ldots, n-i-1\}$.

The pair $(V, \varphi)$ is a conical chart of $x$ and the filtered space $L$ is a link of $x$.

The pseudomanifold $X$ is a classical pseudomanifold when $X_{n-1}=X_{n-2}$, that is , when the codimension one strata do not appear.

In this framework the notion of boundary appears as follows (see [10, 15]).

Definition 1.6. A $n$-dimensional $\partial$-pseudomanifold is a pair $(X, \partial X)$, where $\partial X \subset X$ is a closed subset, together with a filtration on $\emptyset \subseteq X_{0} \subseteq \cdots \subseteq X_{n-1} \subsetneq X_{n}=X$ such that

(a) $X \backslash \partial X$, with the induced filtration $(X \backslash \partial X)_{i}=X_{i} \backslash \partial X$, is an $n$-dimensional pseudomanifold, 
(b) the subset $\partial X$, with the induced filtration $(\partial X)_{i-1}=\partial X \cap X_{i}$, is an $(n-1)$ dimensional pseudomanifold,

(c) the subset $\partial X$ has an open collar neighborhood in $X$, that is, a neighborhood $N$ with a homeomorphism

$$
b: N \rightarrow \partial X \times] 0,1]
$$

preserving the filtrations of Definition 1.2 and sending $\partial X$ to $\partial X \times\{1\}$ by $x \mapsto(x, 1)$.

Notice that $X_{k} \cap \partial X \neq \emptyset$ implies $k>0$. The subset $\partial X$ is called the boundary of $X$. We will often abuse notation by referring to the " $\partial$ - pseudomanifold X", leaving $\partial X$ tacit.

1.7 Refinement of $X$. The local structure of a $\partial$-pseudomanifold $X$ can be described as follows. A point of $X \backslash \partial X$ possesses a conical chart $(V, \varphi)$ in the sense of Definition 1.5. A point $x \in \partial X \cap\left(X_{k} \backslash X_{k-1}\right)$ possesses a conical chart $(V, \varphi)$ in the following sense:

(i) $V \subset X$ is an open neighborhood of $x$, endowed with the induced filtration,

(ii) $\left.\left.\varphi: V \rightarrow \mathbb{R}^{n-1} \times\right] 0,1\right]$ is a homeomorphism with $\varphi(x)=(0,1)($ case $k=n)$,

(iii) $\left.\left.\varphi: V \rightarrow \mathbb{R}^{k-1} \times \stackrel{\circ}{\mathrm{c}} L \times\right] 0,1\right]$ is a stratified homeomorphism, where $L$ is a $(n-k)$ compact pseudomanifold, and $\varphi(x)=(0, \mathrm{v}, 1)$ ( case $k \in\{1, \ldots n-1\})$.

When $\partial X \neq \emptyset$ the space $X$ is not a pseudomanifold (see item (iii)) but it always is a stratified space (this comes directly from from [1, Proposition A.22], (a), (b) and (c)).

Next results show how to refine the stratification of $X$, by considering on $\partial X$ its natural stratification (b), in order to obtain on $X$ a pseudomanifold structure, denoted by $X^{\natural}$. We find this construction on [9] in the category of PL-pseudomanifolds. This procedure is schematized by the following picture:

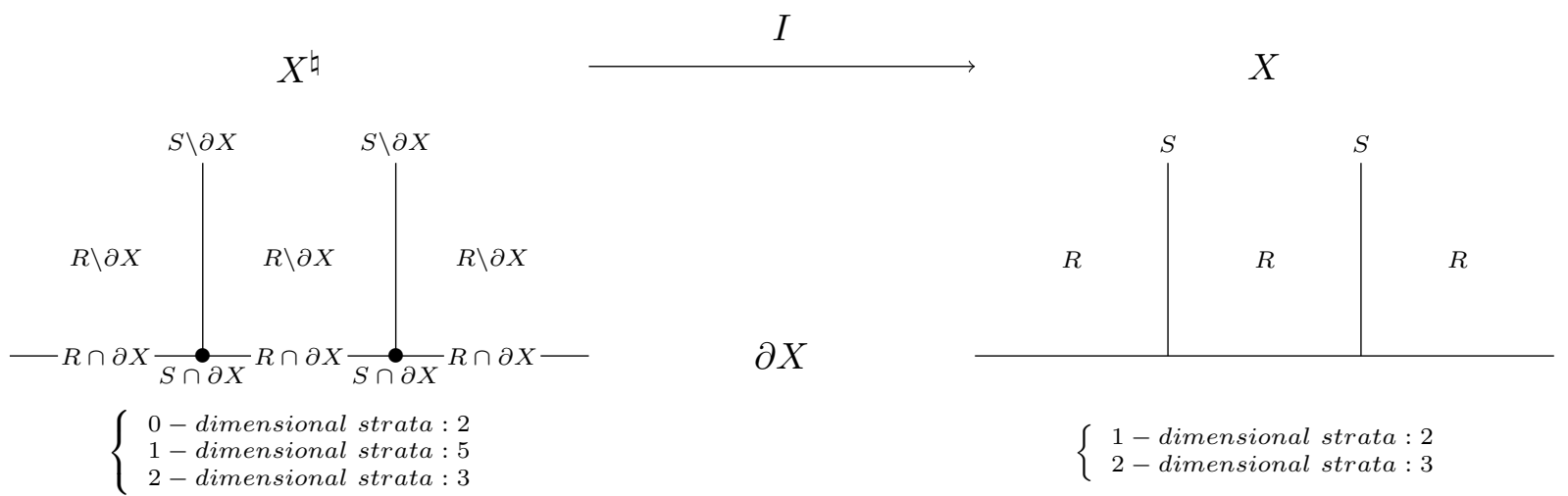

Proposition \& Definition 1.8. Let $X$ be an n-dimensional $\partial$-pseudomanifold. The filtered espace $X_{0}^{\natural} \subset \cdots \subset X_{n}^{\natural}=X^{\natural}$, defined by

$$
X_{k}^{\natural}=X_{k} \cup\left(X_{k+1} \cap \partial X\right),
$$

is an $n$-dimensional pseudomanifold 1 , called the refinement of $X$. The identity $I: X^{\natural} \rightarrow$ $X$ is a stratified map.

\footnotetext{
${ }^{1}$ Here, $X_{n+1}=X$.
} 
Proof. Let us prove that $X^{\natural}$ is an $n$-dimensional pseudomanifold. It is a local question. We proceed by induction on $n$. If $n=0$ then $X^{\natural}=X$ is clearly an $n$-dimensional pseudomanifold.

Since the restriction of both filtrations (that of $X$ and that of $X^{\natural}$ ) to $X^{\natural} \backslash \partial X=$ $X \backslash \partial X$ are equal, then it suffices to consider a point $x \in \partial X$ and construct a conical neighborhood in the sense of 1.7 .

Using the open collar neighborhood $N$, we can suppose $X=B \times] 0,1]$, where $B$ is a $(n-1)$ - pseudomanifold. The filtration on $X$ is the product filtration $\left.\left.X_{i}=B_{i-1} \times\right] 0,1\right]$.

The point $x$ is of the form $(b, 1)$. Now, we can reduce $B$ by considering a conical neighborhood of $b$ in $B$. We distinguish two cases.

(i) $\left.\left.X=\mathbb{R}^{n-1} \times\right] 0,1\right]$. Here, the point $x$ is $(0,1)$. The filtration defining $X^{\natural}$ becomes:

$$
\left.\left.X_{0}^{\natural}=\ldots=X_{n-2}^{\natural}=\emptyset \subset X_{n-1}^{\natural}=\mathbb{R}^{n-1} \times\{1\} \subset X_{n}^{\natural}=\mathbb{R}^{n-1} \times\right] 0,1\right] .
$$

Then $\mathbb{R}^{n-1} \times c\{0\}$ is a conical neighborhood of $x$ in $X^{\natural}$.

(ii) $\left.\left.X=\mathbb{R}^{k} \times \stackrel{\circ}{\mathrm{c}} L \times\right] 0,1\right]$ where $L$ is a $(n-k-2)$-dimensional compact stratified pseudomanifold, with $k \in\{0, \ldots, n-2\}$. Here, the point $x$ is of the form $(0, \mathrm{v}, 1)$, where $\mathrm{v}$ is the apex of $\stackrel{\circ}{c} L$. The refinement $X^{\natural}$ is the product filtration $\left.\left.\mathbb{R}^{k} \times(\stackrel{\circ}{\mathrm{c}} L \times] 0,1\right]\right)^{\natural}$. Without loss of generality, we can suppose $k=0$, that is, $X=\stackrel{\circ}{\circ} L \times] 0,1]$.

Since $L$ is a $(n-2)$-dimensional compact pseudomanifold then the closed cone $c L$ is a $(n-1)$-dimensional $\partial$-pseudomanifold with $\partial c L=L$ and, by induction hypothesis, $(c L)^{\natural}$ is a $(n-1)$-dimensional pseudomanifold. We end the proof if we find a stratified homeomorphism

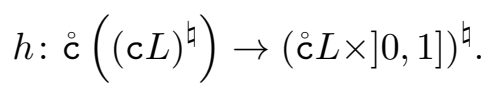

The involved filtrations are

$$
\begin{aligned}
(\{\mathrm{v}\} \times\{1\}) \subset \cdots \subset & \left.\left.\left.\left.\left(\left(\stackrel{\circ}{\mathrm{c}} L_{r-2} \times\right] 0,1\right]\right) \cup\left(\stackrel{\circ}{\mathrm{c}} L_{r-1} \times\{1\}\right)\right) \subset \cdots \subset(\stackrel{\circ}{\mathrm{c}} L \times] 0,1\right]\right), \text { and } \\
& \{\mathrm{w}\} \subset \stackrel{\circ}{\mathrm{c}}\left(\mathrm{c} L_{r-2} \cup L_{r-1}\right) \subset \cdots \subset \stackrel{\circ}{ }(\mathrm{c} L),
\end{aligned}
$$

for $r \in\{1, \ldots, n-1\}$, where $\mathrm{v}$ is the apex of the cones $\stackrel{\circ}{\mathrm{c}} L$ and $\mathrm{c} L$ and $\mathrm{w}$ is the apex of the cone $\stackrel{\circ}{c}(\mathrm{c} L)$. This homeomorphism is defined by

$$
h([[a, x], y])= \begin{cases}([a, 2 x y], 1-y) & \text { si } x \leq 1 / 2 \\ ([a, y], 1-2 y(1-x)) & \text { si } x \geq 1 / 2 .\end{cases}
$$

We verify that previous filtrations are preserved. We proceed in three steps.

+ The restriction

$$
h: \stackrel{\circ}{\mathrm{c}} L \rightarrow \stackrel{\circ}{\mathrm{c}} L \times\{1\},
$$

is given by $h([[a, 1], y])=([a, y], 1)$. Since both induced filtrations are the same: the cone filtration of ${ }^{\circ} L$, then we get that $h$ is a stratified homeomorphism.

+ The restriction

$$
h: \stackrel{\circ}{\mathrm{c}}\{\mathrm{v}\} \rightarrow\{\mathrm{v}\} \times] 0,1],
$$

is given by $h([[a, 0], y])=([a, 0], 1-y)$. Both filtrations have just one singular point: $\{\mathrm{w}\}$ and $(\mathrm{v}, 1)$ respectively. Since $h(\mathrm{w})=(\mathrm{v}, 1)$ then $h$ is a stratified homeomorphism. 
+ The restriction

$$
h: \stackrel{\circ}{\mathrm{c}}(\mathrm{c} L) \backslash \stackrel{\circ}{\mathrm{c}}(\{\mathrm{w}\} \cup L) \rightarrow \backslash(\stackrel{\circ}{\mathrm{c}} L \times] 0,1])(\{\mathrm{v}\} \times] 0,1] \cup \stackrel{\circ}{\mathrm{c}} L \times\{1\})
$$

In fact, this homeomorphism becomes the map $h: L \times] 0,1[\times] 0,1[\rightarrow L \times] 0,1[\times] 0,1[$, defined by

$$
h(a, x, y)= \begin{cases}(a, 1-y, 2 x y) & \text { si } x \leq 1 / 2 \\ (a, 1-2 y(1-x), y) & \text { si } x \geq 1 / 2 .\end{cases}
$$

The maps $h$ is clearly a stratified homeomorphism.

Let us prove the second part of the Proposition. There are two possibilities for a stratum $T \in \mathcal{S}_{X^{\natural}}$ :

- $T=S \backslash \partial X$ with $S \in \mathcal{S}_{X}$ and $\operatorname{codim}_{X^{\natural}} T=\operatorname{codim}_{X} S$, or

- $T=(S \cap \partial X)_{c c}$ with $S \in \mathcal{S}_{X}$ and $\operatorname{codim}_{X^{\natural}} T=\operatorname{codim}_{X} S+1$.

In both cases, $I(T) \subset S$ and $\operatorname{codim}_{X} S \leq \operatorname{codim}_{X^{\natural}} T$. So, $I$ is a stratified map.

Remark 1.9. The stratification of $X^{\natural}$ is given by

$$
\mathcal{S}_{X^{\natural}}=\left\{S \backslash \partial X / S \in \mathcal{S}_{X}\right\} \cup\left\{(S \cap \partial X)_{c c} \neq \emptyset / S \in \mathcal{S}_{X}\right\}
$$

Notice that $X^{\natural}$ and $X$ are equal as topological spaces. They are different as filtered spaces except if $\partial X=\emptyset$. Stratifications induced on $\partial X$ from the two filtered spaces $X, X^{\natural}$ are the same. For this reason, we also write $\partial X \subset X^{\natural}$. We have $X^{\natural} \backslash \partial X=X \backslash \partial X$ as filtered spaces.

\section{TAME intersection homology}

The Lefschetz duality establishes an isomorphism between the blown-up intersection cohomology and the tame intersection homology. In this section, we present the second notion (see [14, 6, 8, 2]). It is an intersection homology where the intersection chains are not included on the singular part of the pseudomanifold. It uses the stratadepending perversities of MacPherson [13] instead the original perversities of Goresky and MacPherson [11]. Working with the original perversities we get that the tame intersection homology is the intersection homology of Goresky and MacPherson [12]

Definition 2.1. Let $X$ be a filtered space. A regular simplex is a continuous map, $\sigma: \Delta \rightarrow X$, where the euclidean simplex $\Delta$ is endowed with a decomposition $\Delta=$ $\Delta_{0} * \Delta_{1} * \cdots * \Delta_{n}$, called $\sigma$-decomposition of $\Delta$, verifying

(a) $\sigma^{-1} X_{i}=\Delta_{0} * \Delta_{1} * \cdots * \Delta_{i}$, for each $i \in\{0, \ldots, n\}$.

(b) $\Delta_{n} \neq \emptyset$.

The perverse degree of $\sigma$ is the $(n+1)$-tuple, $\|\sigma\|=\left(\|\sigma\|_{0}, \ldots,\|\sigma\|_{n}\right)$, where $\|\sigma\|_{i}=$ $\operatorname{dim} \sigma^{-1}\left(X_{n-i}\right)=\operatorname{dim}\left(\Delta_{0} * \cdots * \Delta_{n-i}\right)$, with the convention $\operatorname{dim} \emptyset=-\infty$.

Given a stratum $S$ of $X$, the perverse degree of $\sigma$ along $S$ is defined by

$$
\|\sigma\|_{S}=\left\{\begin{array}{cc}
-\infty, & \text { if } S \cap \operatorname{Im} \sigma=\emptyset \\
\|\sigma\|_{\operatorname{codim} S} & \text { if } S \cap \operatorname{Im} \sigma \neq \emptyset
\end{array}\right.
$$

We set $\mathfrak{d} \Delta$ the regular part of the chain $\partial \Delta$. We define the cochain $\mathfrak{d} \sigma$ by $\sigma \circ \mathfrak{d}$. Notice that $\mathfrak{d}^{2}=0$. We denote by $\mathfrak{C}_{*}(X ; R)$ the chain complex generated by the regular simplices of $X$, endowed with the differential $\mathfrak{d}$. 
Definition 2.2. A perversity on a filtered space $X$ is a map $\bar{p}: \mathcal{S}_{X} \rightarrow \mathbb{Z} \cup\{ \pm \infty\}$ taking the value 0 on the regular strata. The pair $(X, \bar{p})$ is called a perverse space.

The top perversity is the perversity defined by $\bar{t}(S)=\operatorname{codim}(S)-2$ on singular strata. The complementary perversity of a perversity $\bar{p}$ is the perversity $D \bar{p}=\bar{t}-\bar{p}$.

Let $f: X \rightarrow Y$ be a stratified map. The plull-back of a perversity $\bar{q}$ of $Y$ is the perversity $f^{*} \bar{q}$ of $X$ defined by $f^{*} \bar{q}(S)=\bar{q}\left(S^{f}\right)$, for each $S \in \mathcal{S}_{X}$.

Remark 2.3. Let $X$ be a stratified space endowed with a perversity $\bar{p}$. Consider a subset $Y \subset X$ whose induced filtration has formal dimension equal to $n$. The pull-back perversity of $\bar{p}$ relatively to the inclusion $1: Y \hookrightarrow X$ is given by $\iota^{*} \bar{p}\left((S \cap Y)_{c c}\right)=\bar{p}(S)$. For the sake of simplicity we shall write $\iota^{*} \bar{p}=\bar{p}$.

Given a topological manifold $M$, with boundary or not, the canonical projection pr: $M \times X \rightarrow X$ is a stratified map. The pull-back perversity $\operatorname{pr}^{*} \bar{p}$ will be also denoted by $\bar{p}$.

Let us suppose that $X$ is compact. A perversity $\bar{p}$ on the cone $\stackrel{\circ}{ } X$ (resp. $\mathrm{c} X$ ) is determinate by a number $\bar{p}(\mathrm{v}) \in \mathbb{Z} \cup\{ \pm \infty\}$ and a perversity on $X$, still denoted by $\bar{p}$. Here $\mathrm{v}$ is the apex of both cones. These perversities are related by $\bar{p}(S \times] 0,1[)=\bar{p}(S)$ (resp. $\bar{p}(S \times] 0,1])=\bar{p}(S))$ for each stratum $S \in \mathcal{S}_{X}$.

Definition 2.4. Consider a perverse space $(X, \bar{p})$. A simplex $\sigma: \Delta \rightarrow X$ is $\bar{p}$-allowable if

$$
\|\sigma\|_{S} \leq \operatorname{dim} \Delta-\operatorname{codim} S+\bar{p}(S),
$$

for any stratum $S$. We shall say that $\sigma$ is a $\bar{p}$-tame simplex if $\sigma$ is also a regular simplex. A chain $c \in \mathfrak{C}^{*}(X ; R)$ is said to be $\bar{p}$-tame if is a linear combination of $\bar{p}$-tame simplices. The chain $c$ is a tame $\bar{p}$-intersection chain if $c$ and $\mathfrak{d} c$ are $\bar{p}$-tame chains.

We define $\mathfrak{C}_{*}^{\bar{p}}(X ; R)$ the complex of $\bar{p}$-tame intersection chains endowed with the differential $\mathfrak{d}$. Its homology $\mathfrak{H}_{*}^{\bar{p}}(X ; R)$ is the tame $\bar{p}$-intersection homology of $X$

Main properties of this homology have been developed in [2, 8]. We have proven in [2] that the homology $\mathfrak{H}_{*}^{\bar{p}}(X ; R)$ coincides with those of [14, 7] (see also [8, Chapter 6]). It is also proved that, in the case where $\bar{p} \leq \bar{t}$, this homology coincides with the original intersection homology [12].

2.5 Relative tame intersection homology. Let $(X, \bar{p})$ be a perverse space. Consider $Y$ be a subset of $X$ endowed with the induced filtration having the same formal dimension. The complex of relative $\bar{p}$-tame chains is the quotient $\mathfrak{C}_{*}^{\bar{p}}(X, Y ; R)=$ $\mathfrak{C}_{*}^{\bar{p}}(X ; R) / \mathfrak{C}_{*}^{\bar{p}}(Y ; R)$. Its homology is the relative tame $\bar{p}$-intersection homology of the pervers pair $(X, Y, \bar{p})$, denoted by $\mathfrak{H}_{*}^{\bar{p}}(X, Y ; R)$. We have the long exact sequence:

$$
\cdots \rightarrow \mathfrak{H}_{k+1}^{\bar{p}}(X, Y ; R) \rightarrow \mathfrak{H}_{k}^{\bar{p}}(Y ; R) \rightarrow \mathfrak{H}_{k}^{\bar{p}}(X ; R) \stackrel{\mathrm{pr}_{*}}{\longrightarrow} \mathfrak{H}_{k}^{\bar{p}}(X, Y ; R) \rightarrow \ldots,
$$

where pr: $\mathfrak{C}_{*}^{\bar{p}}(X ; R) \rightarrow \mathfrak{C}_{*}^{\bar{p}}(X, Y ; R)$ is the canonical projection

2.6 Perversities relating $X$ and $X^{\natural}$. One of the two key points for establishing our proof of the Lefschetz duality is the understanding of the relationship between the tame intersection homology and the blown-up intersection cohomology of $X$ and $X^{\natural}$. This is done by using a particular perversity on $X^{\natural}$. 
We consider a $\partial$-pseudomanifold $X$ endowed with a perversity $\bar{p}$. We fix a partition of the boundary $\partial X=\partial_{1} X \sqcup \partial_{2} X$ in two families of connected components. Given a perversity $\bar{p}$ over $X$ we define the perversity $\bar{P}$ on $X^{\natural}$ in the following way (see (44)):

$$
\bar{P}=\left\{\begin{array}{cl}
\infty & \text { on the strata included in } \partial_{1} X \backslash \Sigma_{X} \\
-\infty & \text { on the strata included in } \partial_{2} X \backslash \Sigma_{X} \\
I^{*} \bar{p} & \text { on the other strata }
\end{array}\right.
$$

$\bar{P}$

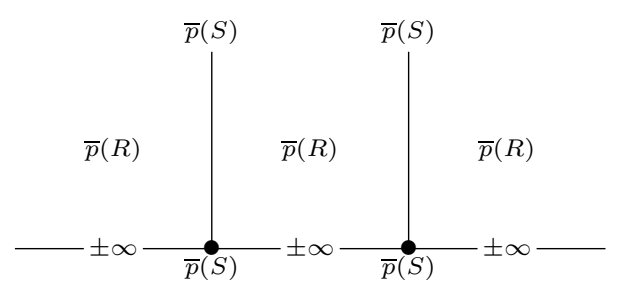

$\bar{p}$

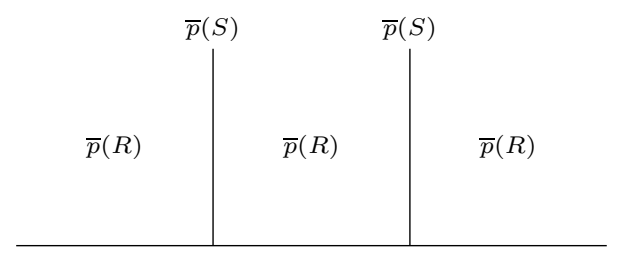

The relationship between the tame intersection homology of $X$ and that of $X^{\natural}$ is given by the following result. We find this result for the intersection homology on [9] in the category of PL-pseudomanifolds

Proposition 2.7. Let $X$ be a $\partial$-pseudomanifold. We consider $\bar{p}$ a perversity on $X$. Let $\partial X=\partial_{1} X \sqcup \partial_{2} X$ a partition of the boundary in two families of connected components. The composition $\operatorname{pr} \circ I_{*}: \mathfrak{C}_{*}^{\bar{P}}\left(X^{\natural} ; R\right) \rightarrow \mathfrak{C}_{*}^{\bar{p}}\left(X, \partial_{1} X ; R\right)$ is a chain map inducing the isomorphism

$$
\mathfrak{I}_{1} \quad: \quad \mathfrak{H}_{*}^{\bar{P}}\left(X^{\natural} ; R\right) \quad \rightarrow \quad \mathfrak{H}_{*}^{\bar{p}}\left(X, \partial_{1} X ; R\right)
$$

Proof. We proceed in two steps.

(I) The operator pr $\circ I_{*}$ is a chain map. The operator $I_{*}: \mathfrak{C}_{*}\left(X^{\natural} ; R\right) \rightarrow \mathfrak{C}_{*}(X ; R)$ is well defined. This comes from [1, Theorem F] and the fact that $I\left(X^{\natural} \backslash \Sigma_{X^{\natural}}\right) \subset X \backslash \Sigma_{X}$, since $I$ is a stratified map (cf. Proposition 1.8). We consider the composition pr $\circ$ $I_{*}: \mathfrak{C}_{*}\left(X^{\natural} ; R\right) \rightarrow \mathfrak{C}_{*}\left(X, \partial_{1} X ; R\right)=\mathfrak{C}_{*}(X ; R) / \mathfrak{C}_{*}\left(\partial_{1} X ; R\right)$. If we prove $\sigma: \Delta \rightarrow X^{\natural}$ is a $\bar{P}$-tame simplex $\Rightarrow \begin{cases}(a) & I_{*}(\sigma): \Delta \rightarrow X \text { is a } \bar{p} \text {-allowable simplex. } \\ (b) & I_{*}(\mathfrak{d} \sigma)-\mathfrak{d} I_{*}(\sigma) \text { is a } \bar{p} \text {-tame chain of } \partial_{1} X,\end{cases}$ then we obtain (I).

(a) The singular strata of $X^{\natural}$ sent to the regular part of $X$ by $I$ are those of the form: $(R \cap \partial X)_{c c}$ where $R$ is a regular stratum of $X$. Outside these strata we have $I^{*} \bar{p}=\bar{P}$. Then, [2, Proposition 3.6, Remark 3.7] says that $I_{*}(\sigma)$ is a $\bar{p}$-allowable simplex. We get (a).

We prove (b). Let $\Delta_{\sigma}=\Delta_{0} * \cdots * \Delta_{n}=\nabla * \Delta_{n}$ be the $\sigma$-decomposition of $\Delta$. If $\left|\Delta_{n}\right| \geq 1$ or $\nabla=\emptyset$ then we have $\mathfrak{d} \sigma=\partial \sigma$ and (b) comes from $I_{*} \circ \partial=\partial \circ I_{*}$. So, we can suppose $\left|\Delta_{n}\right|=0$ and $\nabla \neq \emptyset$. Let $\sigma^{\prime}: \nabla \rightarrow X^{\natural}$ be the restriction of $\sigma$. From $I_{*} \circ \partial=\partial \circ I_{*}$ we get $I_{*}(\mathfrak{d} \sigma)-\mathfrak{d} I_{*}(\sigma)=(-1)^{|\Delta|} I_{*}\left(\sigma^{\prime}\right)$. Condition (b) becomes

$I_{*}\left(\sigma^{\prime}\right)$ is included on $\Sigma_{X}$, or is a $\bar{p}$-tame simplex of $\partial_{1} X$. 
We can suppose that the filtered simplex $I_{*}\left(\sigma^{\prime}\right): \nabla \rightarrow X$ is regular and prove that $I_{*}\left(\sigma^{\prime}\right)$ is a $\bar{p}$-allowable simplex of $\partial_{1} X$.

Consider $\left\{\mathrm{S}_{0} \prec \cdots \prec \mathrm{S}_{a}\right\}$ the family of strata of $\mathcal{S}_{X^{\natural}}$ meeting $\operatorname{Im} \sigma$. Since the simplices $\sigma$ and $I_{*}\left(\sigma^{\prime}\right)$ are regular, then $\mathrm{S}_{a}=(R \backslash \partial X)_{c c}$ and $\mathrm{S}_{a-1}=\left(R \cap \partial_{1} X\right)_{c c}$ or $\left(R \cap \partial_{2} X\right)_{c c}$, for a regular stratum $R \in \mathcal{S}_{X}$.

If $\mathrm{S}_{a-1}=\left(R \cap \partial_{2} X\right)_{c c}$ the $\bar{P}$-allowability of $\sigma$ gives

$$
0 \leq \operatorname{dim} \nabla=\|\sigma\|_{S_{a-1}} \leq \operatorname{dim} \Delta-\operatorname{codim}_{X^{\natural}} \mathrm{S}_{a-1}+\bar{P}\left(\mathrm{~S}_{a-1}\right)=-\infty,
$$

which is impossible. So, we get $\mathrm{S}_{a-1}=\left(R \cap \partial_{1} X\right)_{c c}$. The family of strata of $\mathcal{S}_{X^{\natural}}$ meeting $\operatorname{Im} \sigma^{\prime}$ is

$$
\mathrm{S}_{0}=\left(S_{0} \cap \partial_{1} X\right)_{c c} \prec \cdots \prec \mathrm{S}_{a-2}=\left(S_{a-2} \cap \partial_{1} X\right)_{c c} \prec \mathrm{S}_{a-1}=\left(R \cap \partial_{1} X\right)_{c c},
$$

where $\left\{S_{0}, \ldots, S_{a-2}\right\}$ are singular strata of $X$. This implies that the simplex $I_{*}\left(\sigma^{\prime}\right)$ lies on $\partial_{1} X$. It remains to prove that $I_{*}\left(\sigma^{\prime}\right): \nabla \rightarrow \partial_{1} X$ is $\bar{p}$-allowable. The family of strata of $\partial_{1} X$ meeting $\operatorname{Im} I_{*}\left(\sigma^{\prime}\right)$ is $\left\{\mathrm{S}_{0}, \ldots, \mathrm{S}_{a-2},\left(R \cap \partial_{1} X\right)_{c c}\right\}$ (cf. (16) ). Consider singular stata, that is $j \in\{0, \ldots, a-2\}$, and set $\ell=\operatorname{codim}_{\partial X} \mathrm{~S}_{j}=\operatorname{codim}_{X^{\natural}} \mathrm{S}_{j}-1$. We have

$$
\begin{aligned}
& \left\|I_{*}\left(\sigma^{\prime}\right)\right\|_{\mathrm{S}_{j}} \quad=\quad\left\|I_{*}\left(\sigma^{\prime}\right)\right\|_{\ell}=\operatorname{dim}\left(\Delta_{0} * \cdots * \Delta_{n-\ell}\right)=\|\sigma\|_{\ell} \\
& \sigma \stackrel{\bar{P} \text {-allowable }}{\leq} \operatorname{dim} \Delta-(\ell+1)+\bar{P}\left(\mathrm{~S}_{j}\right)=\operatorname{dim} \nabla-\ell-\bar{p}\left(\mathrm{~S}_{j}\right) \\
& \stackrel{\text { Rem. }}{=} .3 \quad \operatorname{dim} \nabla-\ell+\bar{p}\left(S_{j}\right) \text {. }
\end{aligned}
$$

This gives (b).

(II) The operator $\operatorname{pr} \circ I_{*}$ is a quasi-isomorphism. We proceed by induction on $\operatorname{dim} X$. Notice that the result is clear if $\operatorname{dim} X=0$ or $\partial X=\emptyset$. Using the open collar neighborhood $N$, the equality $X^{\natural} \backslash \partial X=X \backslash \partial X$ (see Remark 1.9) and the Mayer-Vietoris sequence [2, Proposition 7.10], we can suppose $X=\partial X \times] 0,1]$. We prove that

$$
\left.\left.\left.\left.\operatorname{pr} \circ I_{*}: \mathfrak{H}_{*}^{\bar{P}}((\partial X \times] 0,1]\right)^{\natural} ; R\right) \rightarrow \mathfrak{H}_{*}^{\bar{p}}(\partial X \times] 0,1\right], \partial_{1} X \times\{1\} ; R\right)
$$

is an isomorphism by using [5, Proposition 2.19]. Let us verify the four properties. Property (i) comes directly from [2, Proposition 7.10]. Property (ii) is verified since the involved chains have compact support. Property (iv) is straightforward. Let us see property (iii).

We have two different cases to study (see1,7): $X=\mathbb{R}^{n-1} \times\left[0,1\left[\right.\right.$ or $\left.\left.X=\mathbb{R}^{i} \times \stackrel{\circ}{\mathrm{c}} L \times\right] 0,1\right]$, $L$ a compact pseudomanifold. The perversity $\bar{p}$ of $X$ is the perversity $\overline{0}$ in the first case. In the second case, the perversity $\bar{p}$ comes from a perversity $\bar{p}$ defined on $\stackrel{\circ}{\mathrm{c}} L$. The boundary $\partial X=\mathbb{R}^{i} \times \stackrel{\circ}{c} L \times\{1\}$ is connected. We have two different cases to study: $\partial X=\partial_{1} X$ or $\partial X=\partial_{2} X$.

Since the $\mathbb{R}^{n-1}, \mathbb{R}^{i}$-factors commute with the operator $\natural$, then it suffices prove that

(j) $\left.\left.\left.\left.\operatorname{pr} \circ I_{*}: \mathfrak{H}_{*}^{\bar{P}}(] 0,1\right]^{\natural} ; R\right) \rightarrow \mathfrak{H}_{*}^{\overline{0}}(] 0,1\right],\{1\} ; R\right)$ is an isomorphism where $\bar{P}(\{1\})=\infty$.

(jj) $\left.\left.\left.\left.I_{*}: \mathfrak{H}_{*}^{\bar{P}}(] 0,1\right]^{\natural} ; R\right) \rightarrow \mathfrak{H}_{*}^{\overline{0}}(] 0,1\right] ; R\right)$ is an isomorphism where $\bar{P}(\{1\})=-\infty$. 
(jjj) $\left.\left.\left.\left.\operatorname{pr} \circ I_{*}: \mathfrak{H}_{*}^{\bar{P}}((\stackrel{\circ}{\mathrm{c}} L \times] 0,1]\right)^{\natural} ; R\right) \rightarrow \mathfrak{H}_{*}^{\bar{p}}(\stackrel{\circ}{\mathrm{c}} L \times] 0,1\right] ; \stackrel{\circ}{\mathrm{c}} L \times\{1\} ; R\right)$ is an isomorphism for the perversity $\bar{P}=\left\{\begin{array}{cl}\infty & \left.\text { on } L \backslash \Sigma_{L} \times\right] 0,1[\times\{1\} \\ I^{*} \bar{p} & \text { on the other strata. }\end{array}\right.$

(jjjj) $\left.\left.\left.\left.I_{*}: \mathfrak{H}_{*}^{\bar{P}}((\stackrel{\circ}{\mathrm{c}} L \times] 0,1]\right)^{\natural} ; R\right) \rightarrow \mathfrak{H}_{*}^{\bar{p}}(\stackrel{\circ}{\mathrm{c}} L \times] 0,1\right] ; R\right)$ is an isomorphism for the perversity $\bar{P}=\left\{\begin{array}{cl}-\infty & \left.\text { on } L \backslash \Sigma_{L} \times\right] 0,1[\times\{1\} \\ I^{*} \bar{p} & \text { on the other strata. }\end{array}\right.$

Let us prove these properties.

(j) The RHS is $\left.\left.H_{*}(] 0,1\right],\{1\} ; R\right)=0$ (see [2, Definition 4.8 and Proposition 5.5]). On the other hand, $] 0,1]^{\natural}$ is the open cone ${ }^{\circ}\{1\}$ where the induced perversity is given by $\bar{P}(\{1\})=\infty$. Form [2, Proposition 7.9] we get $\left.\left.\mathfrak{H}_{*}^{\bar{P}}(] 0,1\right]^{\natural} ; R\right)=0$.

(jj) The RHS is $\left.\left.\left.H_{*}(10,1] ; R\right)=H_{0}(] 0,1\right] ; R\right)=R$. On the other hand, since the induced perversity is given by $\bar{P}(\{1\})=-\infty$ then, from [2, Proposition 7.9], we get $\left.\left.\mathfrak{H}_{*}^{\bar{P}}(] 0,1\right]^{\natural} ; R\right)=H_{*}(\{1\} ; R)=R$.

(jjj) We know from (51) and 2, Proposition 7.9, Corollaire 7.8] that the RHS is 0 . We have that $\left.\left.h_{*}: \mathfrak{H}_{*}^{h^{*} \bar{P}}\left(\stackrel{\circ}{\mathrm{c}}\left((\mathrm{c} L)^{\natural}\right) ; R\right) \rightarrow \mathfrak{H}_{*}^{\bar{P}}((\stackrel{\circ}{\mathrm{c}} L \times] 0,1]\right)^{\natural} ; R\right)$ is an isomorphism since $h$ is a stratified homeomorphism. Since $h^{*} \bar{P}(\{\mathrm{w}\})=\bar{P}(\{\mathrm{v}\} \times\{1\})=\bar{p}(\{\mathrm{v}\})$ we get $\mathfrak{H}_{*}^{h^{*} \bar{P}}\left(\stackrel{\circ}{\mathrm{c}}\left((\mathrm{c} L)^{\natural}\right) ; R\right)=\mathfrak{H}_{\leq \bar{P}(\{\mathrm{v}\} \times\{1\})}^{\bar{P}}\left((\mathrm{c} L)^{\natural} ; R\right) \stackrel{\text { induction }}{=} \mathfrak{H}_{\leq \bar{p}(\{\mathrm{v}\})}^{\bar{P}}(\mathrm{c} L, L \times\{1\} ; R)=0$ from (5) and [2, Proposition 7.9, Corollaire 7.8].

(jjjj) Let us consider the diagram of filtered spaces:

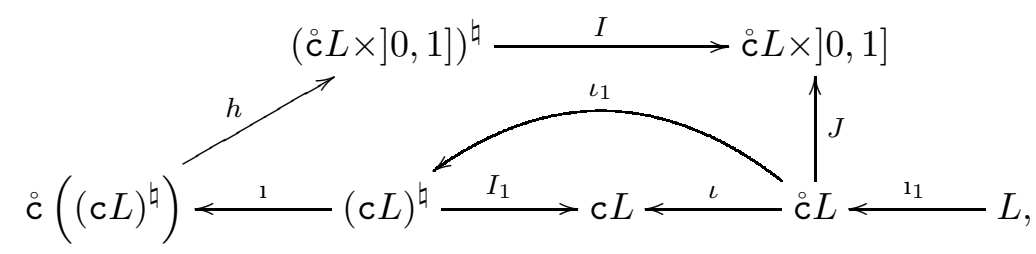

where $I, I_{1}$ are defined in Proposition 1.8, $h$ is defined by (3) $, \iota, \iota_{1}$ are inclusions, $1(x)=1_{1}(x)=[x, 1 / 2]$ and $J(x)=(x, 1 / 2)$. We have the equalities $\iota=I_{1} \circ \iota_{1}$ and $I \circ h \circ 1 \circ \iota_{1} \circ 1_{1}=J \circ 1_{1}$.

The notation $\bar{p}$ designes a perversity on: $\stackrel{\circ}{\mathrm{c}} L \times] 0,1[, \stackrel{\circ}{\mathrm{c}} L \times] 0,1], \stackrel{\circ}{\mathrm{c}} L, \mathrm{c} L$ and $L$ (see Remark 2.3). There are two refined perversities, denoted by $\bar{P}$, on $(\mathrm{c} L)^{\natural}$ and $\left.\left.(\stackrel{\circ}{c} L \times] 0,1\right]\right)^{\natural}$ 
respectively. We also have the perversity $h^{*} \bar{P}$ on $\left.\stackrel{\circ}{(}(\mathrm{c} L)^{\natural}\right)$. They are characterized by:

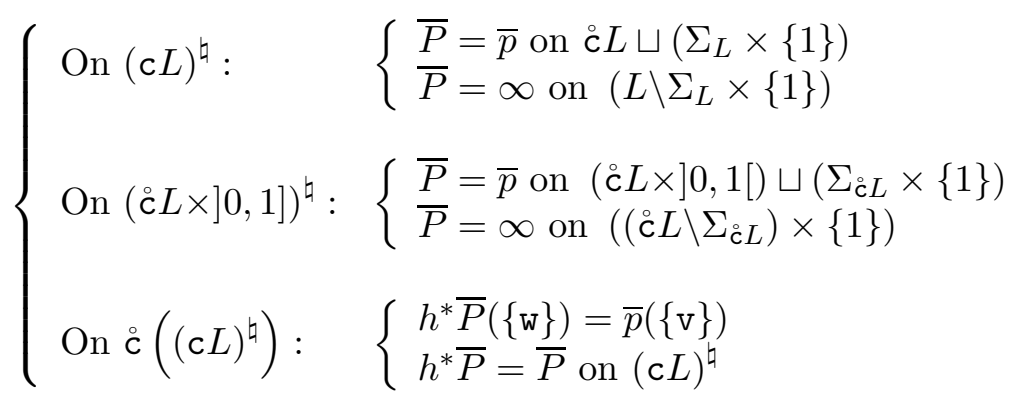

(see Remark 2.3). We have

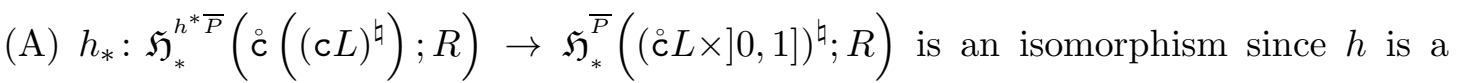
stratified homeomorphism.

(B) $1_{*}: \mathfrak{H}_{\leq \bar{p}(\{\mathrm{v}\})}^{\overline{\bar{P}}}\left((\mathrm{c} L)^{\natural} ; R\right)=\mathfrak{H}_{\leq \bar{p}(\{\mathrm{v}\})}^{h^{*} \bar{p}}\left((\mathrm{c} L)^{\natural} ; R\right) \rightarrow \mathfrak{H}_{*}^{h^{*} \bar{P}}\left(\stackrel{\circ}{(}\left((\mathrm{c} L)^{\natural}\right) ; R\right)$ is an isomorphism since (8) and [2, Proposition 7.9].

(C) $I_{1, *}: \mathfrak{H}_{*}^{\bar{P}}\left((\mathrm{c} L)^{\natural} ; R\right) \rightarrow \mathfrak{H}_{*}^{\bar{p}}(\mathrm{c} L ; R)$ is an isomorphism by induction hypothesis since we have $\operatorname{dim} \mathrm{c} L<\operatorname{dim} X$.

(D) $\iota_{*}: \mathfrak{H}_{*}^{\bar{p}}(\stackrel{\circ}{\mathrm{c}} L ; R) \rightarrow \mathfrak{H}_{*}^{\bar{p}}(\mathrm{c} L ; R)$ is an isomorphism as follows. Consider the open covering $\{U=c L \backslash(L \times\{1\})=\stackrel{c}{*}, V=\mathrm{c} L \backslash\{\mathrm{v}\}=L \times] 0,1]\}$ of $\mathrm{c} L$. From [2, Corollaire 7.8] we know that that the inclusion $U \cap V=L \times] 0,1[\hookrightarrow L \times] 0,1]=V$ induces an isomorphism in homology. Mayer-Vietoris (see [2, Proposition 7.10] ) gives the claim.

(E) $\left.\left.J_{*}: \mathfrak{H}_{*}^{\bar{p}}(\stackrel{\circ}{\mathrm{c}} L ; R) \rightarrow \mathfrak{H}_{*}^{\bar{p}}(\stackrel{\circ}{\mathrm{c}} L \times] 0,1\right] ; R\right)$ is an isomorphism by 2, Corollaire 7.8].

(F) $1_{1, *}: \mathfrak{H}_{\leq \bar{p}(\{v\})}^{\bar{p}}(L ; R) \rightarrow \mathfrak{H}_{*}^{\bar{p}}(\stackrel{\circ}{\mathrm{c}} L ; R)$ is an isomorphism by [2, Proposition 7.9].

We conclude that $\left.\left.\left.\left.I_{*}: \mathfrak{H}_{*}^{\bar{P}}((\stackrel{\circ}{c} L \times] 0,1]\right)^{\natural} ; R\right) \rightarrow \mathfrak{H}_{*}^{\bar{p}}(\stackrel{\circ}{c} L \times] 0,1\right] ; R\right)$ is an isomorphism.

\section{Blown-UP COHOMOLOGY}

The cohomology involved in the Lefschetz Duality is the blown-up cohomology, introduced in [3, 5]. We consider in this section a filtered space $X$.

Let $N_{*}(\Delta)$ and $N^{*}(\Delta)$ denote the simplicial chain and cochain complexes of an euclidean simplex $\Delta$, with coefficients in $R$.

Definition 3.1. The blown-up complex of $X$ with coefficients in $R, \widetilde{N}^{*}(X ; R)$, is the cochain complex formed by the elements $\omega$, associating to any regular simplex, $\sigma: \Delta_{0} *$ $\cdots * \Delta_{n} \rightarrow X$, an element $\omega_{\sigma} \in \widetilde{N}^{*}(\Delta)=N^{*}\left(\mathrm{c} \Delta_{0}\right) \otimes \cdots \otimes N^{*}\left(\mathrm{c} \Delta_{n-1}\right) \otimes N^{*}\left(\Delta_{n}\right)$, compatible with regular faces. The differential $\delta \omega \in \widetilde{N}^{*}(X ; R)$ is defined by $(\delta \omega)_{\sigma}=$ $\delta\left(\omega_{\sigma}\right)$.

Definition 3.2. Let $\Delta_{0} * \cdots * \Delta_{n}$ be a regular simplex. Consider $\ell \in\{1, \ldots, n\}$. Associated to any chain $\xi_{1} \otimes \cdots \otimes \xi_{n} \in \widetilde{N}_{*}\left(\mathcal{H}_{\ell}\right)=N_{*}\left(\mathrm{c} \Delta_{0}\right) \otimes \cdots \otimes N_{*}\left(\mathrm{c} \Delta_{n-\ell-1}\right) \otimes$ $N_{*}\left(\Delta_{n-\ell}\right) \otimes N_{*}\left(\mathrm{c} \Delta_{n-\ell+1}\right) \otimes \cdots \otimes N_{*}\left(\mathrm{c} \Delta_{n-1}\right) \otimes N_{*}\left(\Delta_{n}\right)$ we define $|\xi|_{>n-\ell}=\left|\xi_{n-\ell+1}\right|+$ 
$\cdots+\left|\xi_{n}\right|$, where $|-|$ means degree. The $\ell$-perverse degree of the cochain $\eta \in \widetilde{N}^{*}(\Delta)$ is is equal to

$$
\|\eta\|_{\ell}=\max \left\{|\xi|_{>n-\ell} / \xi \in \widetilde{N}_{*}\left(\mathcal{H}_{\ell}\right) \text { and } \eta(\xi) \neq 0\right\} .
$$

By convention, we set $\max \emptyset=-\infty$.

Definition 3.3. Let $\omega$ a cochain of $\widetilde{N}^{*}(X ; R)$. The perverse degree of $\omega$ along of a singular stratum, $S \in \mathcal{S}$, is equal to

$$
\|\omega\|_{S}=\sup \left\{\left\|\omega_{\sigma}\right\|_{\operatorname{codim} S} \mid \sigma: \Delta \rightarrow X \text { regular with } \sigma(\Delta) \cap S \neq \emptyset\right\} .
$$

By convention, we set $\sup \emptyset=-\infty$. We denote by $\|\omega\|$ the map associating to any singular stratum $S$ of $X$ the element $\|\omega\|_{S}$ and 0 to a regular stratum.

Definition 3.4. Let $(X, \bar{p})$ be a perverse space. A cochain $\omega \in \widetilde{N}^{*}(X ; R)$ is $\bar{p}$-allowable if $\|\omega\| \leq \bar{p}$. A cochain $\omega$ is a $\bar{p}$-intersection cochain if $\omega$ and its coboundary, $\delta \omega$, are $\bar{p}$ allowable. We denote by $\widetilde{N}_{\bar{p}}^{*}(X ; R)$ the complex of $\bar{p}$-intersection cochains and $\mathscr{H}_{*}^{\bar{p}}(X ; R)$ its homology, called blown-up $\bar{p}$-intersection cohomology of $X$ with coefficients in $R$, for the perversity $\bar{p}$.

Let $\mathcal{U}$ be an open covering of $X$. The complex $\widetilde{N}_{\bar{p}}^{*, \mathcal{U}}(X ; R)$ is defined as before excepted that a cochain $\omega \in \widetilde{N}_{\bar{p}}^{*, \mathcal{U}}(X ; R)$ is defined only on the regular simplices $\sigma: \Delta \rightarrow X$ whose image is contained on an element of $\mathcal{U}$.

3.1. Relative blown-up intersection cohomology. Let $(X, \bar{p})$ be a perverse space. Consider $Y$ be a subset of $X$ endowed with the induced filtration and the induced perversity. We suppose $\operatorname{dim} X=\operatorname{dim} Y$. The complex of relative $\bar{p}$-intersection cochains is the direct $\operatorname{sum} \widetilde{N}_{\bar{p}}^{*}(X, Y ; R)=\widetilde{N}_{\bar{p}}^{*}(X ; R) \oplus \widetilde{N}_{\bar{p}}^{*-1}(Y ; R)$, endowed with the derivative $D(\alpha, \beta)=\left(d \alpha, 1^{*} \alpha-d \beta\right)$. Its homology is the relative blown-up intersection cohomology of the perverse pair $(X, Y, \bar{p})$, denoted by $\mathscr{H}_{\bar{p}}^{*}(X, Y ; R)$. We have the long exact sequence associated to the perverse pair $(X, Y, \bar{p})$ :

$$
\ldots \rightarrow \mathscr{H}_{\bar{p}}^{i}(X ; R) \stackrel{\mathrm{I}^{*}}{\rightarrow} \mathscr{H}_{\bar{p}}^{i}(Y ; R) \stackrel{j^{*}}{\rightarrow} \mathscr{H}_{\bar{p}}^{i+1}(X, Y ; R) \stackrel{\mathrm{pr}^{*}}{\longrightarrow} \mathscr{H}_{\bar{p}}^{i+1}(X ; R) \rightarrow \ldots,
$$

where pr: $\widetilde{N}_{\bar{p}}^{*+1}(X, Y ; R) \rightarrow \widetilde{N}_{\bar{p}}^{*}(X ; R)$ is defined by $\operatorname{pr}(\alpha, \beta)=\alpha$ and $j: \widetilde{N}_{\bar{p}}^{*}(Y ; R) \rightarrow$ $\widetilde{N}_{\bar{p}}^{*}(X, Y ; R)$ is defined by $j(\beta)=(0, \beta)$.

Lemma 3.5. Suppose $X$ is a $\partial$-pseudomanifold. Let $Y$ be a union of connected components of the boundary $\partial X$. We consider the open covering $\left.\mathcal{U}=\left\{U=b^{-1}(Y \times] 0,1\right]\right), V=$ $\left.\left.\left.X \backslash b^{-1}(Y \times] 1 / 4,1\right]\right)\right\}$ of $X$. The relative blown-up $\bar{p}$-intersection cohomology of $(X, Y)$ can be computed with the complex

$$
\underline{N}_{\bar{p}}^{*, \mathcal{U}}(X, Y ; R)=\left\{\omega \in \widetilde{N}_{\bar{p}}^{*, \mathcal{U}}(X ; R) / 1^{*} \omega=0\right\} .
$$

Proof. The result is clear when $Y=\emptyset$. Let us suppose $Y \neq \emptyset$. We have seen in 3 , Corollary 9.8] that the restriction $\rho_{\mathcal{U}}: \widetilde{N}_{\bar{p}}^{*}(X ; R) \rightarrow \widetilde{N}_{\bar{p}}^{*, \mathcal{U}}(X ; R)$ induces an isomorphism in homology. So, the restriction $\rho_{\mathcal{U}} \oplus \mathrm{id}: \widetilde{N}_{\bar{p}}^{*}(X, Y ; R) \rightarrow \widetilde{N}_{\bar{p}}^{*, \mathcal{U}}(X, Y ; R)=\widetilde{N}_{\bar{p}}^{*, \mathcal{U}}(X ; R) \oplus$ $\tilde{N}_{\bar{p}}^{*-1}(Y ; R)$ also induces an isomorphism in homology. So, it suffices to prove that 
the inclusion $J: \widetilde{N}_{\bar{p}}^{*, \mathcal{U}}(X, Y ; R) \rightarrow \widetilde{N}_{\bar{p}}^{*, \mathcal{U}}(X, Y ; R)$, defined by $J(\alpha)=(\alpha, 0)$, induces an isomorphism in homology.

Consider $\left.\left.h: N_{Y}=b^{-1}(Y \times] 0,1\right]\right) \rightarrow Y$ the map defined by $h(x)=y$ with $b(x)=(y, t)$. Notice that $h=$ id on $Y$. Consider $a:[0,1] \rightarrow \mathbb{R}$ a continuous map verifying $a \equiv 0$ on $[0,1 / 4]$ and $a \equiv 1$ on $[3 / 4,1]$. Define the continous function $f: X \rightarrow \mathbb{R}$ by $f \equiv 0$ on $\left.\left.X \backslash b^{-1}(Y \times] 1 / 4,1\right]\right)$ and $f\left(b^{-1}(x, t)\right)=a(t)$ on $N_{Y}$. We consider the associated cochain $\tilde{f} \in \widetilde{N}_{\overline{0}}^{0}(X ; R)$ verifying $\tilde{f} \equiv 1$ on $Y$ and $\tilde{f} \equiv 0$ on $\left.\left.X \backslash b^{-1}(Y \times] 1 / 4,1\right]\right)$ (cf. [3, Definition $10.2])$.

If $\beta \in \widetilde{N}_{\bar{p}}^{*}(Y ; R)$ the cochain $\tilde{f} \smile h^{*} \beta$ lives in $\widetilde{N}_{\bar{p}}^{*}\left(N_{Y} ; R\right)$ (see [3, Section 4]) for the definition of cup product $\smile)$. It can be extend it to $\widetilde{N}_{\bar{p}}^{*, \mathcal{U}}(X ; R)$ by 0 since it vanishes on $\left.\left.b^{-1}(Y \times] 0,1 / 4\right]\right)$. It will be denoted also by $\tilde{f} \smile h^{*} \beta \in \widetilde{N}_{\bar{p}}^{*, \mathcal{U}}(X ; R)$. Notice that $1^{*}\left(\tilde{f} \smile h^{*} \beta\right)=\beta$.

Let us prove that $J$ induces an isomorphism in cohomology. Consider a cycle $(\alpha, \beta) \in$ $\tilde{N}_{\bar{p}}^{*, \mathcal{U}}(X, Y ; R)$. It is homologous to $(\alpha, \beta)-D\left(\tilde{f} \smile h^{*} \beta, 0\right)=\left(\alpha-\delta\left(\tilde{f} \smile h^{*} \beta\right), 0\right)=$ $J\left(\alpha-\delta\left(\tilde{f} \smile h^{*} \beta\right)\right)$ with $1^{*}\left(\alpha-\delta\left(\tilde{f} \smile h^{*} \beta\right)\right)=1^{*} \alpha-\delta \beta=0$ and $\delta\left(\alpha-\delta\left(\tilde{f} \smile h^{*} \beta\right)\right)=0$. This gives that $J^{*}$ is an epimorphism. Consider now a cycle $\alpha \in \underline{N}_{\bar{p}}^{*, \mathcal{U}}(X, Y ; R)$ and $(\gamma, \eta) \in \widetilde{N}_{\bar{p}}^{*-1, \mathcal{U}}(X, Y ; R)$ with $D(\gamma, \eta)=J(\alpha)$. As before, $\gamma-\delta(\tilde{f} \smile \eta) \in \underline{\tilde{N}}_{\bar{p}}^{*, \mathcal{U}}(X, Y ; R)$ and $\delta(\gamma-\delta(\tilde{f} \smile \eta))=\alpha$. This gives that $J^{*}$ is an monomorphism.

The relationship between the blown-up intersection homology of $X$ and that of $X^{\natural}$ is given by the following result.

Proposition 3.6. Let $X$ be a paracompact and separable $\partial$-pseudomanifold. We consider $\bar{p}$ a perversity on $X$. Let $\partial X=\partial_{1} X \sqcup \partial_{2} X$ a partition of the boundary in two families of connected components. Consider $\left.\left.\left.\left.\mathcal{U}=\left\{b^{-1}\left(\partial_{2} X \times\right] 0,1\right]\right), X \backslash b^{-1}\left(\partial_{2} X \times\right] 1 / 4,1\right]\right)\right\}$ an open covering of $X$ and $\mathcal{V}$ the induced open covering of $X^{\natural}$ by $I$. The operator $I^{*}: \underline{\widetilde{N}}_{\bar{p}}^{*, \mathcal{U}}\left(X, \partial_{2} X ; R\right) \rightarrow \widetilde{N}_{\bar{P}}^{*, \mathcal{V}}\left(X^{\natural} ; R\right)$ is a chain map inducing the isomorphism

$$
\mathfrak{I}_{2} \quad: \quad \mathscr{H}_{\bar{p}}^{*}\left(X, \partial_{2} X ; R\right) \rightarrow \mathscr{H}_{\bar{P}}^{*}\left(X^{\natural} ; R\right)
$$

Proof. We proceed in two steps.

(I) The operator $I^{*}$ is a chain map. Since $I$ is a stratified map the induced map $I^{*}: \widetilde{N}^{*}(X ; R) \rightarrow \widetilde{N}^{*}\left(X^{\natural} ; R\right)$ is a well defined chain map (cf. [3, Definition 8.2] and so $I^{*}: \underline{\tilde{N}}_{\bar{p}}^{*, \mathcal{U}}\left(X, \partial_{2} X ; R\right) \rightarrow \widetilde{N}^{*, \mathcal{V}}\left(X^{\natural} ; R\right)$. Let us prove that $I^{*}: \underline{\tilde{N}}_{\bar{p}}^{*, \mathcal{U}}\left(X, \partial_{2} X ; R\right) \rightarrow$ $\tilde{N}_{\bar{P}}^{*, \mathcal{V}}\left(X^{\natural} ; R\right)$ is well defined. Recall that $1: \partial_{2} X \hookrightarrow X$ denotes the natural inclusion.

It suffices to consider $\alpha \in \widetilde{N}_{\bar{p}}^{*, \mathcal{U}}(X ; R)$, with $1^{*} \alpha=0$, and $S \in \mathcal{S}_{X^{\natural}}$ a singular stratum and to prove: $\left\|I^{*} \omega\right\|_{S} \leq \bar{P}(S)$. We have $\left\|I^{*} \omega\right\|_{S} \leq\|\omega\|_{S^{I}}$ (cf. [3, Theorem A]) and $I^{*} \bar{p}(S) \leq \bar{P}(S)$ if $S$ is not of the form $\left(R \cap \partial_{2} X\right)_{c c}$ for a regular stratum $R$ of $X$. So, it remains to prove

$$
\left\|I^{*} \omega\right\|_{\left(R \cap \partial_{2} X\right)_{c c}}=-\infty .
$$

Let $\sigma: \Delta \rightarrow X^{\natural}$ be a regular simplex with $\operatorname{Im} \sigma \cap\left(R \cap \partial_{2} X\right)_{c c} \neq \emptyset$ and $\operatorname{Im} \sigma \subset$

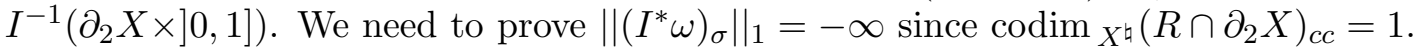


The family of strata of $X^{\natural}$ meeting $\operatorname{Im} \sigma$ is necessarily of the form

$$
\left(S_{0} \cap \partial_{2} X\right)_{c c} \prec \cdots \prec\left(S_{b-1} \cap \partial_{2} X\right)_{c c} \prec\left(R \cap \partial_{2} X\right)_{c c} \prec R \backslash \partial X,
$$

where $S_{0} \prec \cdots \prec S_{b-1}$ are singular strata of $X$ preceding $R$. So, if $\Delta_{0} * \cdots * \Delta_{n}$ is the $\sigma$-decomposition of $\Delta$ we we get that the $I_{*}(\sigma)$-decomposition is the elementary amalgamation $\mathcal{A}_{\sigma}: \Delta_{0} * \cdots * \Delta_{n} \rightarrow \Delta_{0} * \cdots * \Delta_{n-2} *\left(\Delta_{n-1} * \Delta_{n}\right)$ (cf. [3, Section 7]). Notice that $\left.\left.\operatorname{Im} I_{*}(\sigma) \subset b^{-1}\left(\partial_{2} X \times\right] 0,1\right]\right)$. So, $\left\|I^{*} \omega\right\|_{\left(R \cap \partial_{2} X\right)_{c c}}=\left\|\mathcal{A}_{\sigma}^{*} \omega_{I_{*}(\sigma)}\right\|_{1}$ (see [3, Definition 8.2]). Following the definition of $\|-\|_{1}$ we need to prove that the restriction of the cochain $\mathcal{A}_{\sigma}^{*} \omega_{I_{*}(\sigma)}$ to $\widetilde{N}_{*}\left(\mathcal{H}_{n-1}\right)=N_{*}\left(\mathrm{c} \Delta_{0}\right) \otimes \cdots \otimes N_{*}\left(\mathrm{c} \Delta_{n-1}\right) \otimes N_{*}\left(\Delta_{n-1}\right) \otimes N_{*}\left(\Delta_{n}\right)$ vanishes (cf. Definition 3.2). Let us consider the commutative diagram:

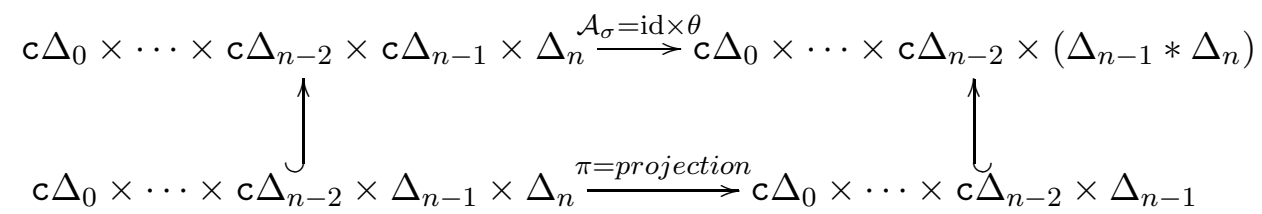

(cf. [3, Definition 7.3]). So, the restriction of $\mathcal{A}_{\sigma}^{*} \omega_{I_{*}(\sigma)}$ to $\widetilde{N}_{*}\left(\mathcal{H}_{n-1}\right)$ is the pull-back $\pi^{*}$ of the restriction of $\omega_{I_{*}(\sigma)}$ to $N_{*}\left(\mathrm{c} \Delta_{0}\right) \otimes \cdots \otimes \mathrm{c} N_{*}\left(\Delta_{n-2}\right) \otimes N_{*}\left(\Delta_{n-1}\right)$. Let us consider the simplex $\sigma^{\prime}: \Delta_{0} * \cdots * \Delta_{n-1} \hookrightarrow \Delta \stackrel{\sigma}{\rightarrow} X^{\natural} \stackrel{I}{\rightarrow} X$. It is a regular simplex whose associated filtration is exactly $\Delta_{0} * \cdots * \Delta_{n-1}$ (cf. (10) ). By compatibility with regular faces, the restriction of $\omega_{I_{*}(\sigma)}$ to $\widetilde{N}_{*}\left(\mathrm{c} \Delta_{0}\right) \otimes \cdots \otimes \widetilde{N}_{*}\left(\mathrm{c} \Delta_{n-2}\right) \times \widetilde{N}_{*}\left(\Delta_{n-1}\right)$ is exactly $\omega_{\sigma^{\prime}}$. Finally, since $\sigma^{\prime}$ is a regular simplex of $\partial_{2} X$ and $1^{*} \omega=0$ then $\omega_{\sigma^{\prime}}=0$.

(II) The operator $I^{*}$ is a quasi-isomorphism. This ends the proof following Lemma3.5, We proceed by induction on the dimension of $X$. Notice that the result is clear if $\operatorname{dim} X=$ 0 or $\partial X=\emptyset$. Using the open collar neighborhood $N$, the equality $X^{\natural} \backslash \partial X=X \backslash \partial X$ (see Remark 1.9) and the Mayer-Vietoris sequence [3, Theorem C], we can suppose $X=\partial X \times] 0,1]$.

Using [3, Proposition 13.2] we prove that

$$
\left.\left.\left.\left.I^{*}: \mathscr{H}_{\bar{p}}^{*}(\partial X \times] 0,1\right], \partial_{2} X ; R\right) \rightarrow \mathscr{H}_{\bar{P}}^{*}((\partial X \times] 0,1]\right)^{\natural} ; R\right)
$$

is an isomorphism. We verify the four properties. Property (i) comes directly from 3 , Theorem A]. Properties (ii) and (iv) are straightforward. Let us see property (iii).

We have two diffrent cases to study (see1,7): $X=\mathbb{R}^{n-1} \times\left[0,1\left[\right.\right.$ or $\left.\left.X=\mathbb{R}^{i} \times \stackrel{\text { c }}{L} \times\right] 0,1\right]$, $L$ being a compact pseudomanifold $L$. The perversity $\bar{p}$ of $X$ is the perversity $\overline{0}$ in the first case. In the second case, the perversity $\bar{p}$ comes in fact from a perversity $\bar{p}$ defined on $\stackrel{\circ}{\mathrm{c}} L$. The boundary $\partial X=\mathbb{R}^{i} \times \stackrel{\circ}{\mathrm{c}} L \times\{1\}$ is connected. We have two different cases $\partial X=\partial_{1} X$ or $\partial X=\partial_{2} X$.

Since the $\mathbb{R}^{n-1}, \mathbb{R}^{i}$-factors commute with the operator $\measuredangle$ then it suffices prove that

(j) $\left.\left.\left.\left.I_{*}: \mathscr{H}_{\bar{p}}^{*}(] 0,1\right],\{1\} ; R\right) \rightarrow \mathscr{H}_{\bar{P}}^{*}(] 0,1\right]^{\natural} ; R\right)$ is an isomorphism where $\bar{P}(\{1\})=-\infty$.

(jj) $\left.\left.\left.\left.I^{*}: \mathscr{H}_{\bar{p}}^{*}(] 0,1\right] ; R\right) \rightarrow \mathfrak{H}_{\bar{P}}^{*}(] 0,1\right]^{\natural} ; R\right)$ is an isomorphism where $\bar{P}(\{1\})=\infty$. 
(jjj) $\left.\left.\left.\left.I^{*}: \mathscr{H}_{\bar{p}}^{*}(\stackrel{\circ}{\mathrm{c}} L \times] 0,1\right] ; \stackrel{\circ}{\mathrm{c}} L \times\{1\} ; R\right) \rightarrow \mathscr{H}_{\bar{P}}^{*}((\stackrel{\circ}{\mathrm{c}} L \times] 0,1]\right)^{\natural} ; R\right)$ is an isomorphism for the perversity $\bar{P}=\left\{\begin{array}{cl}-\infty & \left.\text { on } L \backslash \Sigma_{L} \times\right] 0,1[\times\{1\} \\ I^{*} \bar{p} & \text { on the other strata. }\end{array}\right.$

$\left.\left.\left.\left.(\mathrm{jjjj}) I_{*}: \mathscr{H}_{\bar{p}}^{*}(\stackrel{\circ}{\mathrm{c} L} \times] 0,1\right] ; R\right) \rightarrow \mathscr{H}_{\bar{P}}^{*}((\stackrel{\circ}{\mathrm{c} L} \times] 0,1]\right)^{\natural} ; R\right)$ is an isomorphism for the perversity $\bar{P}=\left\{\begin{array}{cl}\infty & \left.\text { on } L \backslash \Sigma_{L} \times\right] 0,1[\times\{1\} \\ I^{*} \bar{p} & \text { on the other strata. }\end{array}\right.$

Let us prove these properties.

(j) The LHS is $\left.\left.H^{*}(] 0,1\right],\{1\} ; R\right)=0$ (cf. [3, 12.5 and Theorem E]). On the other hand, ] $0,1]^{\natural}$ is the open cone $\check{c}\{\mathrm{v}\}$ where the induced perversity is given by $\bar{p}(\{\mathrm{v}\})=-\infty$. From [3, Theorem E] we get $\left.\left.\mathscr{H}_{\bar{P}}^{*}(] 0,1\right]^{\natural} ; R\right)=0$.

(jj) The LHS is $\left.\left.H^{*}(10,1] ; R\right)=H^{0}(10,1] ; R\right)=R$. On the other hand, since the induced perversity is given by $\bar{p}(\{\mathrm{v}\})=\infty$ then, from [3, Theorem E], we get $\left.\left.\mathscr{H}_{\bar{P}}^{*}(] 0,1\right]^{\natural} ; R\right)=$ $H^{*}(\{\mathrm{v}\} ; R)=R$.

(jjj) We know from (9) and [3, Theorems D,E] that the LHS is 0 . We have that

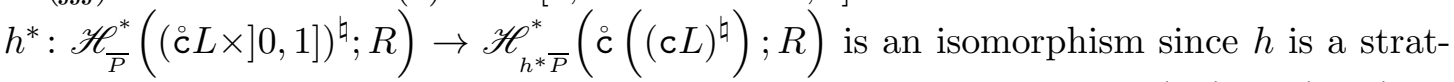
ified homeomorphism. Using (며) and [3], Theorem E] we get $\mathscr{H}_{h^{*} \bar{P}}\left(\mathrm{c}\left((\mathrm{c} L)^{\natural}\right) ; R\right)=$ $\mathscr{H}_{\bar{P}}^{\leq \bar{p}\{v\}}\left((\mathrm{c} L)^{\natural} ; R\right) \stackrel{\text { induction }}{=} \mathscr{H}_{\bar{q}}^{\leq \bar{p}(\{v\})}(\mathrm{c} L, L \times\{1\} ; R)=0$ from [3, Proposition 12.7].

(jjjj) Let us consider the commutative diagram of filtered spaces (77). We have

(A) $\left.\left.h^{*}: \mathscr{H}_{\bar{P}}^{*}((\stackrel{\circ}{\mathrm{c} L} \times] 0,1]\right)^{\natural} ; R\right) \rightarrow \mathscr{H}_{h^{*} \bar{P}}\left(\stackrel{\circ}{\mathrm{c}}\left((\mathrm{c} L)^{\natural}\right) ; R\right)$ is an isomorphism since $h$ is a stratified homeomorphism.

(B) $1^{*}: \mathscr{H}_{h^{*} \bar{P}}^{*}\left(\stackrel{\circ}{(}\left((\mathrm{c} L)^{\natural}\right) ; R\right) \rightarrow \mathscr{H}_{h^{*} \bar{P}}^{\leq \bar{p}(\mathrm{v})}\left((\mathrm{c} L)^{\natural} ; R\right)=\mathscr{H}_{\bar{P}}^{\leq \bar{p}(\mathrm{v})}\left((\mathrm{c} L)^{\natural} ; R\right)$ is an isomorphism since (8) and [3, Theorem E].

(C) $I_{1}^{*}: \mathscr{H}_{\bar{p}}^{*}(\mathrm{c} L ; R) \rightarrow \mathscr{H}_{\bar{P}}^{*}\left((\mathrm{c} L)^{\natural} ; R\right)$ is an isomorphism by induction hypothesis since we have $\operatorname{dim} \mathrm{c} L<\operatorname{dim} X$.

(D) $\iota^{*}: \mathscr{H}_{\bar{p}}^{*}(\mathrm{c} L ; R) \rightarrow \mathscr{H}_{\bar{p}}^{*}(\stackrel{\circ}{\mathrm{c}} L ; R)$ is an isomorphism with the same proof of Proposition 2.7 (D) using [3, Theorems C,D].

(E) $\left.\left.J^{*}: \mathscr{H}_{\bar{p}}^{*}(\stackrel{\circ}{\mathrm{c}} L \times] 0,1\right] ; R\right) \rightarrow \mathscr{H}_{\bar{p}}^{*}(\stackrel{\circ}{\mathrm{c}} L ; R)$ is an isomorphism by [3, Theorem D].

(F) $1_{1}^{*}: \mathscr{H}_{\bar{p}}^{*}(\stackrel{\circ}{\mathrm{c}} L ; R) \rightarrow \mathscr{H}_{\bar{p}}^{\leq \bar{p}(\mathrm{v})}(L ; R)$ is an isomorphism by [무, Theorem E].

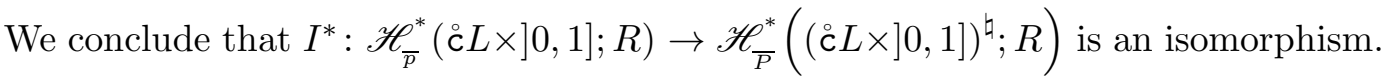

\section{Lefschetz Duality}

We prove the main result of this work: the Lefschetz Duality. First, we present the orientation issues, following [8]. 
4.1 Orientation and fundamental class. An $n$-dimensional $\partial$-pseudomanifold $X$ is $R$-orientable if the pseudomanifold $X \backslash \partial X$ is $R$-orientable. When $X$ is compact, associated to an $R$-orientation, there exists the fundamental class $\Gamma_{X} \in H_{n}^{\overline{0}}(X, \partial X ; R)$ (see [8, Theorem 8.3.3]). We fix a relative cycle $\gamma_{X} \in \mathfrak{C}_{n}^{\overline{0}}(X ; R)$ representing this class, that is, $\mathfrak{d} \gamma_{X} \in \mathfrak{C}_{n-1}^{\overline{0}}(\partial X ; R)$ and $\Gamma_{X}=\left[\operatorname{pr}\left(\gamma_{X}\right)\right]$, where pr: $\mathfrak{C}_{*}^{\bar{p}}(X ; R) \rightarrow \mathfrak{C}_{*}^{\bar{p}}(X, \partial X ; R)$ is the canonical projection.

The operator pr $\circ I_{*}: \mathfrak{H}_{*}^{\overline{0}}\left(X^{\natural} ; R\right) \rightarrow \mathfrak{H}_{*}^{0}(X, \partial X ; R)$ is a well defined morphism (direct consequence of Proposition 2.7). We can relate the fundamental classes of $X^{\natural}$ and $(X, \partial X)$ as follows.

Proposition 4.2. Let $X$ be an $n$-dimensional $R$-oriented compact $\partial$-pseudomanifold. Then, the pseudomanifold $X^{\natural}$ is R-orientable. Moreover, if $\Gamma_{X^{\natural}} \in \mathfrak{H}_{n}^{\overline{0}}\left(X^{\natural} ; R\right)$ is the fundamental class of $X^{\natural}$ then $\operatorname{pr} \circ I_{*}\left(\Gamma_{X^{\natural}}\right)$ is the fundamental class of $X$.

Proof. The orientability of $X^{\natural}$ comes from the fact that $X^{\natural} \backslash \partial X=X \backslash \partial X$, as stratified pseudomanifolds. The orientation sheaf $\mathcal{O}^{\overline{0}}$ over $X \backslash \partial X=X^{\natural} \backslash \partial X$ is therefore the same. We write $\mathfrak{o}^{\overline{0}}$ the associated global section. For the second part, we consider a point $x \in X \backslash \partial X$ and we prove that the restriction of $\operatorname{pr} \circ I_{*}\left(\Gamma_{X^{\natural}}\right)$ to $\mathfrak{H}_{*}^{\bar{\sigma}}(X, X \backslash\{x\} ; R)=$ $\mathfrak{H}_{*}^{\overline{0}}(X \backslash \partial X,(X \backslash \partial X) \backslash\{x\} ; R)$ [2, Corollaire 7.12] is $\mathfrak{o}^{\overline{0}}(x)$ [8, Theorem 8.3.3]. This comes from the fact that the restriction of $\left[\gamma_{X^{\natural}}\right]$ to $\mathfrak{H}_{*}^{\overline{0}}\left(X^{\natural}, X^{\natural} \backslash\{x\}\right)=\mathfrak{H}_{*}^{\overline{0}}\left(X^{\natural} \backslash \partial X,\left(X^{\natural} \backslash \partial X\right) \backslash\{x\}\right)$ [2, Corollaire 7.12] is $\mathfrak{o}^{\overline{0}}(x)$ (cf. [8, Theorem 8.1.15]).

4.3 Duality operator $\mathcal{D}_{X}$. We consider $\partial X=\partial_{1} X \sqcup \partial_{2} X$ a partition of the boundary in two families of connected components. Using the subdivision operator and the associated homotopy operator of [2, Proposition 7.10] we can suppose that $\gamma_{X} \in$ $\mathfrak{C}_{n}^{\overline{0}}(U ; R)+\mathfrak{C}_{n}^{\overline{0}}(V ; R)$, where the covering $\mathcal{U}=\{U, V\}$ is defined in Lemma 3.5, relatively to $Y=\partial_{2} X$.

We fix a perversity $\bar{p}$ on $X$. The cup product with the fundamental class is the homomorphism

$$
\mathcal{D}_{X}: \mathscr{H}_{\bar{p}}^{*}\left(X, \partial_{2} X ; R\right) \rightarrow \mathfrak{H}_{n-*}^{\bar{p}}\left(X, \partial_{1} X ; R\right)
$$

defined by

$$
\mathcal{D}_{X}([\alpha])=\left[\operatorname{pr}\left(\alpha \frown \gamma_{X}\right)\right] .
$$

where $\alpha$ is a cycle of $\underline{\tilde{N}}_{\bar{p}}^{*, \mathcal{U}}\left(X, \partial_{2} X ; R\right)$ and pr: $\mathfrak{C}_{*}^{\bar{p}}(X ; R) \rightarrow \mathfrak{C}_{*}^{\bar{p}}\left(X, \partial_{1} X ; R\right)$ is the canonical projection. This operator is well defined since $\gamma_{X} \in \mathfrak{C}_{n}^{\overline{0}}(U ; R)+\mathfrak{C}_{n}^{\overline{0}}(V ; R), \operatorname{pr}\left(\alpha \frown \eta_{1}\right)=0$ and $\operatorname{pr}\left(\alpha \frown \eta_{2}\right)=0\left(\alpha\right.$ vanishes on $\left.\partial_{2} X\right)$, where $\partial \gamma_{X}=\eta_{1}+\eta_{2}$ is the canonical decomposition relatively to $\partial X=\partial_{1} X \sqcup \partial_{2} X$.

Theorem A. Let $X$ be an n-dimensional oriented compact $\partial$-pseudomanifold. Consider a perversity $\bar{p}$ on $X$. Let $\partial X=\partial_{1} X \cup \partial_{2} X$ a partition of the boundary in two families of connected components. The cup product with the fundamental class induces the isomorphism

$$
\mathcal{D}_{X}: \mathscr{H}_{\bar{p}}^{*}\left(X, \partial_{2} X ; R\right) \rightarrow \mathfrak{H}_{n-*}^{\bar{p}}\left(X, \partial_{1} X ; R\right)
$$


Proof. We consider the diagram

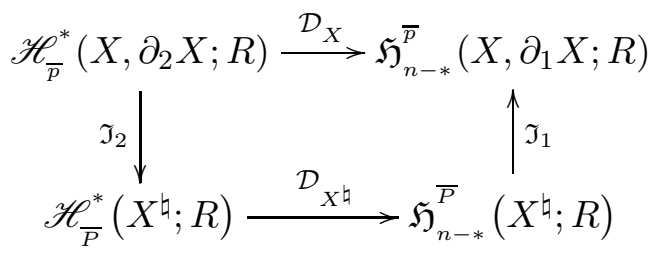

where the vertical arrows and the bottom arrow are isomorphisms (Proposition 2.7, Proposition [3.6 and [5, Theorem B]). Notice that $X$ is paracompact and separable. The second assertion comes from the fact that $X$ is recovered by a finite number of conical charts (see 1.7) and that each of them is separable. This is clear for the charts of type (ii). For those of type (iii) it suffice to apply induction on $L$.

We end the proof if we show the diagram commutes.

We consider $\gamma^{\natural} \in \mathfrak{C}_{n}^{\overline{0}}\left(X^{\natural} ; R\right)$ a generator of the fundamental class $\Gamma^{\natural}$ of $X^{\natural}$, see Proposition 4.2. This Proposition also gives $\xi \in \mathfrak{C}_{n+1}^{\overline{0}}(X ; R)$ and $\xi_{k} \in \mathfrak{C}_{n}^{\overline{0}}\left(\partial_{k} X ; R\right), k=1,2$, with $I_{*} \gamma_{X^{\natural}}-\gamma_{X}=\xi_{1}+\xi_{2}+\partial \xi$. Let $\alpha$ be a cycle of $\underline{\widetilde{N}}_{\bar{p}}^{*, \mathcal{U}}\left(X, \partial_{2} X ; R\right)$. Notice that $\alpha \frown \xi_{1} \in \mathfrak{C}_{n-*}^{\bar{p}}\left(\partial_{1} X ; R\right)$ and that $\alpha \frown \xi_{2}=0$ since $\alpha$ vanishes on $\partial_{2} X$. Then

$$
\begin{aligned}
& \mathfrak{I}_{1} \mathcal{D}_{X^{\natural}} \mathfrak{I}_{2}([\alpha])=\mathfrak{I}_{1} \mathcal{D}_{X^{\natural}}\left(\left[I^{*} \alpha\right]\right)=\mathfrak{I}_{1}\left(\left[I^{*} \alpha \frown \gamma_{X^{\natural}}\right]\right)=\left[\operatorname{pr}\left(I_{*}\left(I^{*} \alpha \frown \gamma_{X^{\natural}}\right)\right)\right] \stackrel{3 \text { Theorem A(3) }}{=} \\
& {\left[\operatorname{pr}\left(\alpha \frown I_{*} \gamma_{X^{\natural}}\right)\right]=\left[\operatorname{pr}\left(\alpha \frown \gamma_{X}+\alpha \frown \xi_{1}+\alpha \frown \xi_{2}+\alpha \frown \partial \xi\right)\right] \quad=} \\
& {\left[\operatorname{pr}\left(\alpha \frown \gamma_{X} \pm \partial(\alpha \frown \xi)\right)\right]=\mathcal{D}_{X}([\alpha]) .}
\end{aligned}
$$

\section{REFERENCES}

[1] D. Chataur, M. Saralegi-Aranguren \& D. Tanré - "Intersection Cohomology. Simplicial blowup and rational homotopy.", ArXiv Mathematics e-prints (2012), To appear in Mem. Amer. Math. Soc.

[2] _ "Intersection homology. General perversities and topological invariance", ArXiv Mathematics e-prints 1602.03009 (2016).

[3] , "Blown-up intersection cohomology", in An alpine bouquet of algebraic topology, Contemp. Math., vol. 708, Amer. Math. Soc., Providence, RI, 2018, p. 45-102.

[4] _ "Blown-up intersection cochains and Deligne's sheaves", ArXiv Mathematics e-prints 1801.02992 (2018).

[5] _ "Poincaré duality with cap products in intersection homology", Adv. Math. 326 (2018), p. 314-351.

[6] G. FRIEDMAN - "Superperverse intersection cohomology: stratification (in)dependence", Math. Z. 252 (2006), no. 1, p. 49-70.

[7] _ "Singular chain intersection homology for traditional and super-perversities", Trans. Amer. Math. Soc. 359 (2007), no. 5, p. 1977-2019 (electronic).

[8] G. FRIEDMAN - "Singular intersection homology", Available at http://faculty.tcu.edu/gfriedman/index.html

[9] G. Friedman \& E. HunsiCKER - "Additivity and non-additivity for perverse signatures", J. Reine Angew. Math. 676 (2013), p. 51-95.

[10] G. Friedman \& J. E. MCClure - "Cup and cap products in intersection (co)homology", Adv. Math. 240 (2013), p. 383-426.

[11] M. Goresky \& R. MacPherson - "Intersection homology theory", Topology 19 (1980), no. 2, p. $135-162$. 
[12] _ "Intersection homology. II", Invent. Math. 72 (1983), no. 1, p. 77-129.

[13] R. MACPherson - "Intersection homology and perverse sheaves", Unpublished AMS Colloquium Lectures, San Francisco (1991).

[14] M. SARAlegi-ArAnguren - "de Rham intersection cohomology for general perversities", Illinois J. Math. 49 (2005), no. 3, p. 737-758 (electronic).

[15] G. Valette - "A Lefschetz duality for intersection homology", Geom. Dedicata 169 (2014), p. 283299.

Laboratoire de Mathématiques de Lens, EA 2462, Université D'Artois, SP18, Rue Jean Souvraz, 62307 Lens Cedex, France

E-mail address: martin.saraleguiaranguren@univ-artois.fr 